\title{
Direct demonstration of transsynaptic degeneration in the human visual system: a comparison of retrograde and anterograde changes
}

\author{
RM BEATTY, AA SADUN, LEH SMITH, JP VONSATTEL, EP RICHARDSON, JR \\ From the CS Kubik Laboratory for Neuropathology, Department of Pathology, Massachusetts General \\ Hospital, the Department of Ophthalmology, Massachusetts Eye and Ear Infirmary, and the Departments of \\ Neurology-Neuropathology, Pathology, and Ophthalmology, Harvard Medical School, Boston, Mass, USA
}

SUMMARY Transneuronal degeneration of retinal ganglion cells was directly demonstrated in a patient who had unilateral removal of the striate cortex forty years prior to necropsy. For comparison, another case is presented showing anterograde transneuronal atrophy forty years after enucleation of one eye.

Transsynaptic retrograde degeneration (TRD) has been described in several neuronal systems. Some of these are: the rabbit mammillary nuclei, ${ }^{1}$ the rat and rabbit ventral tegmental tract, ${ }^{1}$ human pyramidal neurons in the motor cortex, ${ }^{1}$ human inferior olivary nuclei, ${ }^{12}$ rat $^{1}$ and human ${ }^{2}$ cerebellum, the avian homologue of the Edinger-Westphal nucleus ${ }^{1}$ and the retinal ganglion cell in human beings, ${ }^{2-7}$ kittens, ${ }^{8}$ puppies, ${ }^{1}$ and monkeys. ${ }^{9-12}$ Observations were made in man as early as 1880.67 These, and those of subsequent authors, have indicated that the severity of TRD was inversely proportional to the age of the subject at the time of injury and directly proportional to the time elapsed following the injury.

Although TRD has been inferred in the human visual system, it has never been directly demonstrated. Previous studies have described the development of optic atrophy following damage to the visual cortex. ${ }^{34611}$ Euzière reported the case of a WorldWar I veteran in whom bilateral optic atrophy was noted 15 years after a traumatic penetrating injury to the right parieto-occipital region. ${ }^{3}$ The description of the optic atrophy (pale, cupped discs), however, and the findings on visual field examination are more typical of glaucoma than anything that would be expected from occipital cortex injury; moreover, the intraocular pressure in this case was $20 \mathrm{~mm} \mathrm{Hg}$. Haddock described the clinical presence of bilateral

Address for reprint requests: RM Beatty, MD, The Children's Hospital Medical Center, Department of Neurosurgery, 300 Longwood Avenue, Boston, Mass 02115, USA.

Received 5 July 1981

Accepted 6 August 1981 optic atrophy in a World-War II veteran six years following a penetrating injury to the left and right occipital regions. ${ }^{4}$ This case was complicated by the presence of papilloedema and other signs of increased intracranial pressure. As in Euzière's case, a direct lesion of the retinal ganglion cells could not be ruled out.

Van Buren described three cases of metastatic cancer involving the occipital regions, with degenerative changes in the lateral geniculate nuclei and appropriate regions of the retinae.11 Papilloedema was present in each of these cases, however, leaving doubt as to whether the ocular abnormalities were the result of direct or of transneuronal degeneration. Haddock's study was based only on clinical observation; Van Buren's investigations included autopsy material. However, neither of these nor previous studies had the benefit of a method which could, with great sensitivity, identify degenerated axons. With such a technique, transsynaptic retrograde degeneration can be demonstrated while general and diffuse anterograde changes can be ruled out.

In the following case reports, paraphenylene diamine (PPD) was used to stain degenerated axons and axon terminals. This technique has been shown to be a highly sensitive and reliable method for identifying degenerated axons in human brain material. ${ }^{15}$ PPD has been shown to stain degenerated axons following lesions that occurred more than five years before death. ${ }^{14}$ The resolution achieved by this technique is better than can be obtained with silver impregnation; it thus allows the identification of very small numbers of fibres. ${ }^{14}$ 


\section{Case materials and methods}

Case 1 In 1941, when the patient was 46 years old, he was evaluated at the Massachusetts General Hospital (MGH) because of adult-onset seizures. There was no history of trauma or infection. The physical and ophthalmologic examinations were normal. Skull radiographs suggested meningioma or vascular malformation in the right occipital area. A craniotomy was done, and a resection was carried out which included a considerable part of the right occipital lobe and adjacent portions of the posterior temporal and lower parietal regions. There was no damage to the vascular supply of the lateral geniculate nucleus. $^{16}$ Pathological examination of the biopsy specimen showed the presence of an angiomatous malformation. Post-operatively, the patient had a complete left homonymous hemianopsia, but was otherwise normal. He was seen again in 1959, and in 1961; the left homonymous hemianopsia was noted on these occasions, but there were no other neurological abnormalities. In 1976, examination by an ophthalmologist documented the left homonymous hemianopsia. Visual acuity was $20 / 30$ in the right eye and $20 / 70$ in the left eye; the optic discs were pale, ocular tension was normal bilaterally. In late December 1980 the patient experienced a transient left sided hemiparesis. In mid-January 1981, he died.

Postmortem examination revealed acute myocardial infarction, bilateral bronchopneumonia, and occult prostatic adenocarcinoma without evidence of metastasis. The brain was fixed in $10 \%$ formalin for 14 days. Grossly, it showed a $4 \times 1.5 \mathrm{~cm}$ cavitated lesion in the right parieto-occipital area. At the coronal level of the foramen of Monro, the diameter of the right optic tract was $1.5 \mathrm{~mm}$ and that of the left optic tract was $2.5 \mathrm{~mm}$. The right optic nerve measured $4.5 \times 2.0 \mathrm{~mm}$, and the left measured $4.0 \times 1.8 \mathrm{~mm}$. Cut sections were dehydrated and embedded in paraffin according to standard hospital protocol. Representative sections were stained with cresyl violet, and haematoxylin and eosin. In addition, three-micron-thick sections were also cut from the paraffin embedded blocks. These sections were mounted and stained with PPD according to the Sadun-Smith method. ${ }^{15}$

Microscopically, there was a small infarct in the right middle frontal gyrus and another in the right putaminal region. Each infarct was judged to be a few weeks old. There was striking asymmetry of the lateral geniculate nuclei (LGN) the right being atrophic as compared with the left (figs 1 and 2). When stained with PPD, degenerated axons appeared as dark brown circular profiles and axon terminals as smaller dots (fig 3). Degenerated axons were seen in the optic radiations above the right lateral geniculate nucleus. Degenerated axons were also seen adjacent to each layer of the right LGN and in the anterior ventral part of the nucleus as the distal portion of the right optic tract. No degenerated axons were seen in the left lateral geniculate nucleus or left optic tract. The optic chiasm contained degenerated axons intermingled with normal axons anteriorly with a complete segregation of degenerated fibres from normal ones posteriorly.

Case 2 This patient sustained a traumatic enucleation of

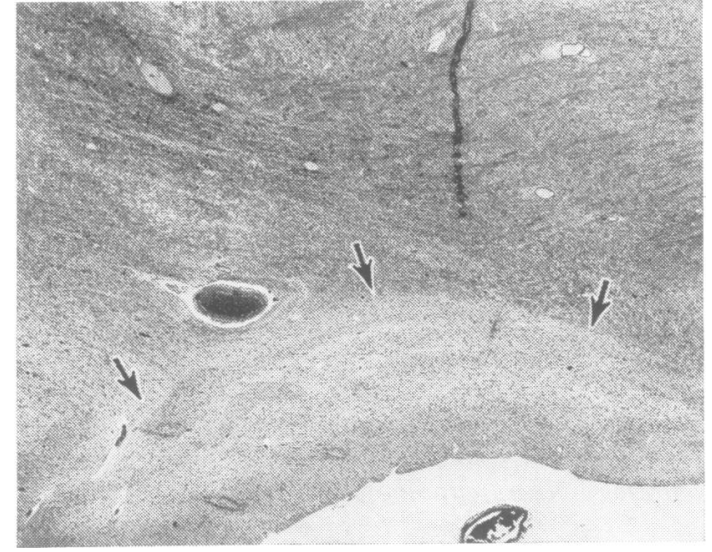

Fig 1 Case 1; right LGN. The arrows outline the atrophied LGN ipsilateral to the occipital lesion. $H$ and E. $\times 14$.

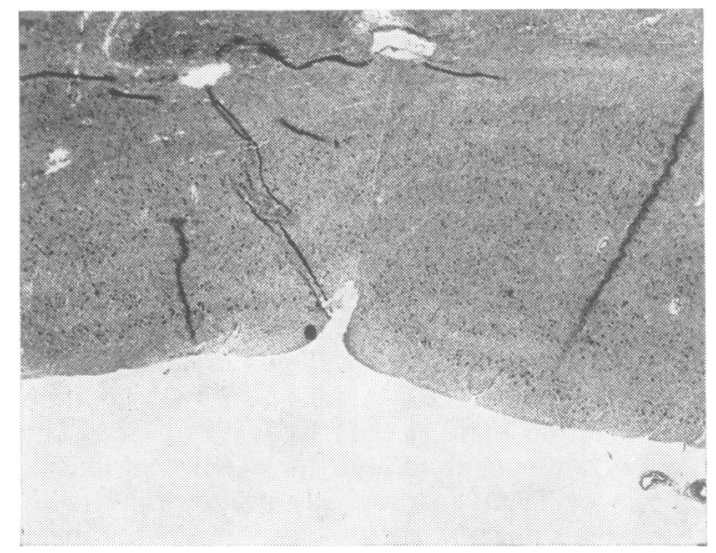

Fig 2 Case 1; left LGN. The normal appearing LGN controlateral to the occipital lesion. $H$ and $E . \times 14$.

the entire right eye in 1941 during World-War II. He was in good health until June 1979, when acute myelogenous leukaemia developed. He died in April 1981. Postmortem examination revealed disseminated evidence of myelogenous leukaemia (liver, spleen, lung, kidney and marrow). No leukaemic involvement was detected in the brain or meninges. The brain was fixed in $10 \%$ formalin for 14 days. The right optic nerve measured $3 \mathrm{~mm}$ in diameter and the left measured $7 \mathrm{~mm}$. Tissue was prepared for microscopic examination in the manner previously described.

Microscopically, the right optic nerve was composed of fibrous and gliotic tissue containing no myelin or macrophages. From the chiasm backward the myelin staining was normal. The right lateral geniculate nucleus, ispilateral to the enucleation, showed marked neuronal loss in laminae 2,3 , and 5 , whereas laminae 1,4 , and 6 were normal. The left lateral geniculate nucleus showed neu- 


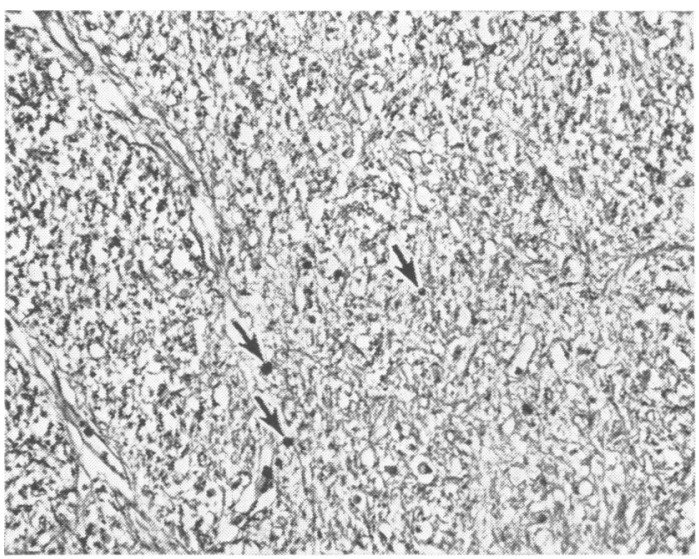

Fig 3 Case 1; right LGN. The arrows point to degenerated axons which are stained with the PPD technique. $\times 250$.

ronal loss in laminae 1,4 , and 6 , while laminae 2,3 , and 5 were normal. The calcarine cortex showed normal cytoarchitecture without atrophic features. A few degenerating axons were seen with PPD in the optic radiations bilaterally. Degenerated axons were seen adjacent to atrophic neurons in layers 2,3 , and 5 in the right lateral geniculate nucleus and adjacent to atrophic cells in layers 1, 4, and 6 in the left lateral geniculate nucleus (fig 4). Both optic tracts showed degenerated axons, but they were found only in the right optic nerve.

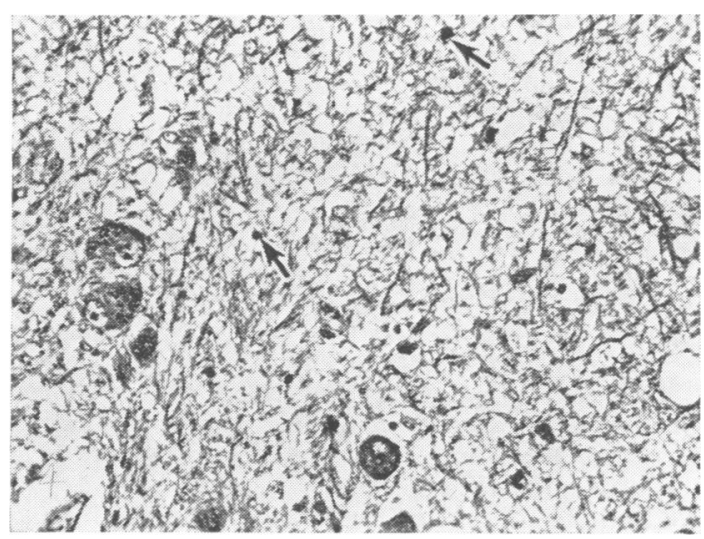

Fig 4 Case 2; right LGN. The arrows point to degenerated axons in lamina two. PPD. $\times 250$.

\section{Discussion}

We have presented the clinical and neuropathological findings in two cases of transneuronal degeneration in the human visual system, occurring many years after the original lesion. In our study of these cases, we have used not only the usual histopathological methods, but, in addition, a technique that makes possible the selective demonstration of degenerating axons - the PPD method. This enabled us to map directly the location of axonal degeneration in the various components of the visual pathways, andmore importantly-to exclude the possibility of damage to these fibre systems resulting from concomitant or adventitious disorders.

In Case 1, an entire right occipital lobe had been surgically removed 40 years before the patient's death; optic atrophy was seen 35 years after the operation. In addition to extensive atrophy of neurons in the right lateral geniculate nucleus, there were degenerated axons, as demonstrated by the PPD method, in the right optic tract, and both optic nerves. No degenerated axons were found in the left lateral geniculate nucleus or left optic tract, thus excluding the possibility of a lesion anterior to the chiasm, which would have resulted in degeneration in the optic nerves and lateral geniculate nuclei bilaterally (fig 5). The findings in this case, therefore,

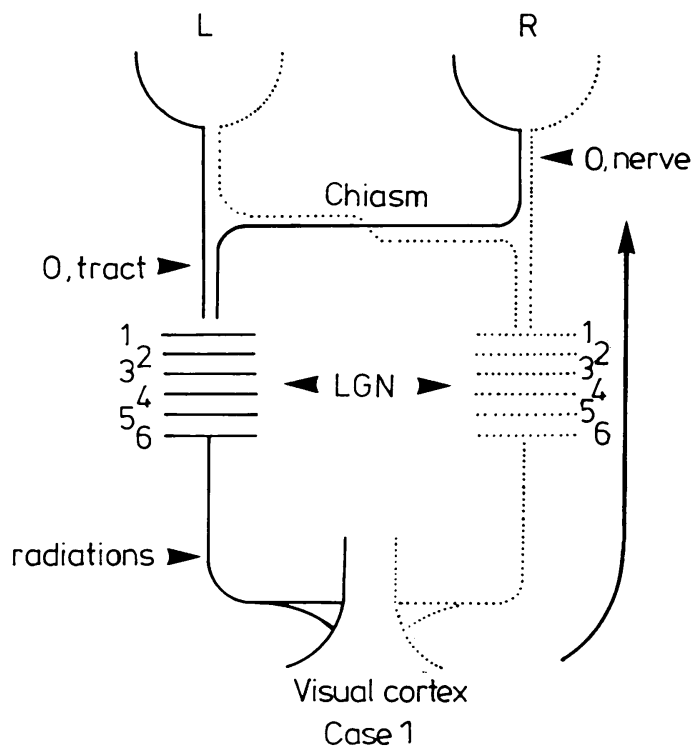

Fig 5 A diagram illustrating Case 1 in which a lesion of the occipital lobe resulted in retrograde degeneration of all six laminae of the $L G N$.

indicate the presence of transneuronal retrograde degeneration 40 years after the removal of one occipital lobe. Case 2 demonstrated the reverse (fig 6). Here there was anterograde degeneration following enucleation of the right eye; degenerated axons were seen in the right optic nerve, both optic tracts, and in both lateral geniculate nuclei in the 


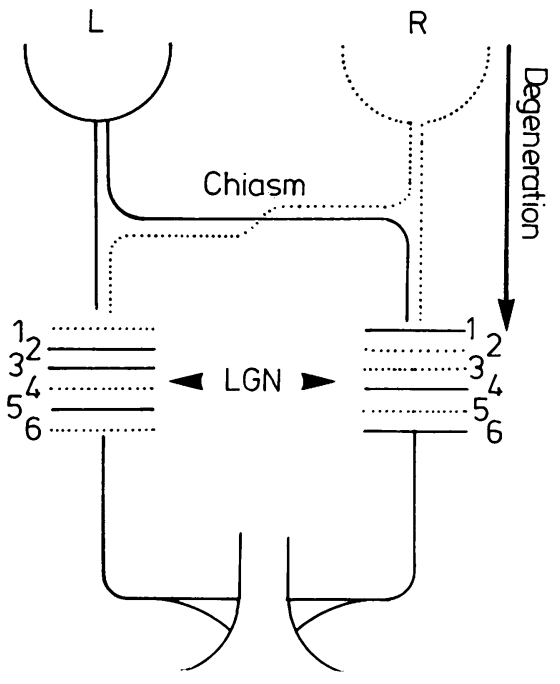

Case 2

Fig 6 A diagram illustrating Case 2 in which a lesion of the optic nerve resulted in anterograde degeneration of each optic tract and atrophy of selective layers (dots) of each LGN.

neuronal laminae corresponding to the missing eye. 5617

The clinical implication of the findings in Case 1 is that after the passage of many years, a unilateral extensive lesion of the visual system in the occipital lobe of an adult can result in the fundiscopic appearance of bilateral optic atrophy. In children, optic atrophy has been seen to occur at shorter intervals following an occipital lesion. ${ }^{6} 712$

The transneuronal changes, both retrograde and anterograde, that were seen in these two cases, were demonstrated directly in necropsy material by means of a staining method that is selective for degenerated axons. We therefore believe that this method now makes it possible to demonstrate histologically in man the transneuronal effects that have more frequently been described in experimental animals.

The authors thank Ms Lovely Templeton for her efforts in typing this manuscript and Ms Carla Wittig for her gracious help in the diagrams.

\section{References}

${ }^{1}$ Cowan WM. Anterograde and Retrograde Transneuronal Degeneration in the Central and Peripheral Nervous System. In: Nauta WJH, Ebbesson SOE, eds. Contemporary Research Methods in Neuroanatomy. Heidelberg: Springer-Verlag, 1970:21751.

${ }^{2}$ Blackwood W. Transsynaptic Degeneration. In: Blackwood W, Corsellis JAN, eds. Greenfield's Neuropathology. Third ed. Chicago: Year Book Med Pub, Inc, 1976:14-15.

${ }^{3}$ Euzière J, Viallefont H, Vidal J. Double atrophie optique et hemianopsie gauche consecutives à une blessure occipitale droite. Arch Soc Sci Med Biol Montpellier 1943;14:212-5.

${ }^{4}$ Haddock JN. Transsynaptic degeneration in the visual system. Arch Neur Psychiat 1950;64:66-73.

${ }^{5}$ Lindenberg R. Neuropathology of Vision. An Atlas. Philadelphia: Lea and Febiger, 1973:336-61.

' Moeli C. Veränderungen des Tractus and Nervus Opticus bei Erkrankungen des Occipitalhirns. Arch Psychiat Nervenkrankh 1981 ;22:73-120.

${ }^{7}$ von Monakow C. Experimentelle und pathologischanatomische Untersuchungen über die optischen Centren und Bahnen. Arch Psychiat Nervenkrankh 1888;20:714-87.

${ }^{8}$ Ganser S. Über die periphere und zentrale Anordnung der Sehnervenfasern und über das Corpus bigeminum anterius. Arch Psychiat Nervenkr 1882;13:341-81.

${ }^{9}$ Matthews MR. Transneuronal cell degeneration in the lateral geniculate nucleus of the macaque monkey. $J$ Anat 1960;94:145-69.

${ }^{10}$ Van Buren JM. The Retinal Ganglion Cell Layer. Springfield: CC Thomas, 1963:102-21.

11 Van Buren JM. Transsynaptic retrograde degeneration in the visual system of primates. J Neurol Neurosurg Psychiatry 1963;26:402-7.

${ }^{12}$ Walsh FB, Hoyt WF. Clinical Neuro-ophthalmology. Vol. I, ed. 3. Baltimore: Williams and Wilkins Co, 1969:624.

${ }^{13}$ Sadun AA, Smith EH, Kenyon KR. The visual pathways in man. A new fiber tracing method. Assoc Res in Vision and Ophthal Abstr 1981.

${ }^{14}$ Smith EH, Sadun AA, Nauta WH, Schneider GE. Retinal projections to the human superior colliculus. A preliminary report. Society for Neurosci 1981 .

${ }^{15}$ Sadun AA. Differential distribution of cortical terminations in the cat red nucleus. Brain Res 1975; 99:145-51.

${ }^{16}$ Polyak S. The Vertebrate Visual System. Chicago: Univ of Chicago Press, 1957:304-5, 606-10.

${ }^{17}$ Carpenter MB. Human Neuroanatomy. Seventh ed. Baltimore: Williams and Wilkins Co, 1976:458. 